La primera intervención, denominada Proyecto 2000, comenzó en 1996 y abarcó los 12 departamentos peruanos con mayor mortalidad materna. El objetivo del Proyecto, financiado por la Agencia de los Estados Unidos para el Desarrollo Internacional (USAID), fue aumentar la proporción de los partos atendidos en centros de salud y de esta forma reducir la mortalidad materna y el número de partos con desenlaces negativos. El proyecto comenzó con la educación y movilización social de la población y llamó mediante los medios de comunicación masiva a que las mujeres dieran a luz en los centros de emergencia obstétrica (CEO). Su principal finalidad fue, sin embargo, mejorar la calidad de los servicios.

La segunda intervención, denominada Programa de Seguro Materno-infantil (SMI), se puso en marcha en 1998. Este programa cubría la mayor parte de los costos de salud de madre hijo, entre ellos el parto en un CEO. Solo las mujeres ubicadas en el quinto más pobre de la población podían participar. Ya en el año 2000, este programa beneficiaba a 50\% de las familias con derecho en las dos regiones en que se realizaron las pruebas piloto y al año siguiente se extendió a todo el país.

Ambos programas seguían estrategias diferentes: mientras que el Proyecto 2000 estaba orientado a brindar una atención de mayor calidad en los distritos de alto riesgo y se centraba en instalaciones específicas de CEO y en las comunidades circundantes, el programa SIM estaba enfocado hacia las familias más pobres del país.

Después de controlar los resultados según el nivel económico de las familias, se comprobó que las madres participantes del Proyecto 2000 estaban más dispuestas a dar a luz en un CEO público que las que no participaban. No obstante, el programa SIM tuvo un mayor impacto directo en el comportamiento de las futuras madres, ya que al reducir los gastos de bolsillo por el uso de los CEO, elevó la proporción de partos en instituciones médicas. El componente de interacción fue insignificante, lo que demuestra que a pesar de que ambos programas estaban dirigidos a la misma población, cada uno funcionó independientemente del otro.

Si bien el Proyecto 2000 mejoró la calidad de la atención médica brindada, no logró aumentar el número de partos realizados en los CEO. Por su parte, el programa SIM tuvo un impacto temporal que duró mientras se mantuvo el apoyo financiero, lo que demuestra que el costo de los servicios era la principal barrera que impedía el uso de los CEO. El principal inconveniente de estas intervenciones subsidiadas es que por lo general no son sostenibles, especialmente en los países pobres.

Aunque las mujeres más pobres se beneficiaron de ambas intervenciones, los factores de riesgo siguieron teniendo su efecto negativo en todos los modelos, lo que demuestra que ninguna de las dos logró reducir significativamente las desigualdades socioeconómicas o étnicas que obstaculizaban el uso de los CEO. (McQuestion MJ, Velásquez A. Evaluating program effects on institutional delivery in Peru. Health Policy. 2006;77(2):221-32.)

\section{Los ingresos nacionales y su relación con la esperanza de vida}

Las críticas a las hipótesis que relacionan los ingresos con la salud han puesto de relieve la falta de datos que confirmen el efecto diferenciado de la desigualdad entre los países ricos y pobres. A pesar de esas críticas, no se ha prestado la debida atención a desarrollar criterios que permitan clasificar más objetivamente los países en ricos y pobres.

En este artículo se analiza si la utilización de criterios alternativos de estratificación de los países puede ayudar a dilucidar el efecto que sobre la esperanza de vida tienen los ingresos y su desigualdad. Además, se examina si han ocurrido cambios significativos en la estabilidad estructural de los diferentes grupos de países.

Para ello se agruparon 107 países según cuatro criterios relacionados con los ingresos y el comercio: 1) ingresos, se consideraron países de ingresos altos cuando el producto interno bruto per cápita (PIB/c) promedio en la década de 1990 fue mayor de US\$ 5000,00 , según lo cual, 54 países se consideraron de ingresos altos y 53 de ingresos bajos; 2) pertenencia a la Organización para la Colaboración y el Desarrollo Económicos (OCDE), se consideró que un país pertenecía a la OCDE si era miembro pleno desde antes de 1990, según lo cual, 23 países eran miembros de esa organización y 84 no lo eran; 3) pertenencia al núcleo del comercio mundial, se consideró que un país pertenecía a este subgrupo si participaba en el comercio de productos de capital intensivo, según lo cual, 29 países pertenecían al núcleo comercial y 78 no pertenecían; y 4) pertenencia a la periferia, se consideraron así los países cuyo papel en el comercio mundial era insignificante, según lo cual, 61 de los países estudiados no pertenecían a la periferia y 46 sí. Toda la información se obtuvo de las bases de datos de las $\mathrm{Na}$ ciones Unidas y del Banco Mundial. Los 23 países que pertenecían a la OCDE tenían ingresos altos, pertenecían al núcleo comercial y no se consideraron periféricos. Se utilizó cada criterio por separado para buscar diferencias significativas entre los diferentes grupos de países según la esperanza de vida en ellos.

El empleo de pruebas de comparación de grupos y del análisis de regresión permitió encontrar 
diferencias significativas en la esperanza de vida, el $\mathrm{PIB} / \mathrm{c}$ y la desigualdad de los ingresos en los dos subgrupos de países de cada una de las cuatro categorías. Las mayores diferencias en cuanto al PIB/c y a la desigualdad de los ingresos se observaron entre los países miembros de la OCDE y los que no eran miembros de esa organización, mientras que la mayor diferencia en cuanto a la esperanza de vida se encontró entre los países considerados periféricos y los no periféricos. El análisis combinado de los datos demostró que los ingresos tienen un efecto directo significativo sobre la esperanza de vida y confirmó el efecto adverso de la desigualdad de los ingresos sobre la esperanza de vida.

No se encontraron diferencias significativas en la esperanza de vida, el PIB/c y la desigualdad de los ingresos ni entre los países de ingresos altos y bajos ni entre los países pertenecientes o no al núcleo comercial.

Estos resultados permiten rechazar la hipótesis de que las diferencias en los ingresos de los países reflejan mejor su estratificación en ricos y pobres y que los efectos negativos de la desigualdad en los ingresos son mayores en los países de ingresos altos. Por el contrario, demuestran que a partir de los patrones comerciales se logra una mejor estratificación de los países y que la desigualdad en los ingresos tiene un efecto adverso sobre la esperanza de vida en países de la periferia. (Moore $S$. Peripherality, income inequality, and life expectancy: revisiting the income inequality hypothesis. Int J Epidemiol. 2006;35(3):623-32.)

\section{Problemas de salud de los adultos mayores en una población de la frontera entre México y los Estados Unidos}

La frontera entre los Estados Unidos de América y México es un área excepcional desde muchos puntos de vista. Además de considerarse una de las regiones más dinámicas del mundo, el frecuente cruce de la frontera influye en la cultura y el estilo de vida de las poblaciones asentadas en su entono. El rápido aumento de la población en la frontera afecta a la infraestructura de la atención sanitaria y representa un reto para los profesionales de la salud y las autoridades encargadas de eliminar las desigualdades de salud y de ofrecer una atención ajustada a las características culturales de esa población, especialmente de los adultos mayores.

En este estudio se analizan los problemas de este grupo de la población en un condado rural de Arizona, Estados Unidos, fronterizo con México. Mediante entrevistas a 29 informantes clave - proveedores de servicios sociales y de salud y líderes comunitarios- y con datos de los archivos públicos se analizaron: 1) el estado de salud de los adultos mayores, 2) sus necesidades de atención sanitaria y su patrón de utilización, 3) la capacidad de la comunidad de satisfacer las necesidades de los adultos mayores y las deficiencias de la atención, 4) la influencia de la cultura en las creencias y prácticas relacionadas con la salud y 5) las necesidades educacionales de los profesionales de la salud relacionadas con el envejecimiento y el cuidado de los adultos mayores.

Los resultados de este estudio indicaron que existen desigualdades que afectan a la salud de los adultos mayores en la zona fronteriza. El principal problema que impide eliminar esas desigualdades es el insuficiente acceso de los adultos mayores a la atención sanitaria en esta región. Esto no solo se debió a problemas financieros, sino también a la insuficiente infraestructura de la comunidad, a las condiciones geográficas, demográficas y socioeconómicas, al poco conocimiento de esta población acerca de la salud, a las particularidades de la cultura dominante, al desconocimiento de la población acerca del envejecimiento y de los problemas específicos relacionados con cruzar la frontera. La diversidad de temas relacionados con la salud de los adultos mayores en esta zona indica que las causas de esas desigualdades son multifactoriales.

El impacto de las diferencias culturales en los conceptos y prácticas relacionados con la salud se manifestó tanto en los pacientes como en los proveedores de los servicios de salud. Por ello, el reto para los proveedores de asistencia sanitaria consiste en desarrollar un sistema competente desde el punto de vista cultural y conocer mejor las particularidades socioculturales de esta comunidad y de sus adultos mayores.

Los adultos mayores que viven en la zona fronteriza constituyen una población especialmente vulnerable. A pesar de que este grupo de la población víctima de la desigualdad ha crecido, el enfoque tradicional de los sistemas de salud ha contribuido a que se desatiendan sus necesidades de salud. La comunidad, los encargados de establecer las políticas y las agencias y proveedores de servicios sociales y de salud deben informarse mejor acerca de los problemas que aquejan a los adultos mayores y preparar adecuadamente al personal para brindar una atención de salud de calidad y sensible al entorno cultural. En este trabajo se propone un marco de acción para desarrollar programas educativos y proyectos de investigación dirigidos a los profesionales de salud encargados de atender a los adultos mayores que viven en esta zona fronteriza. (Guo G, Phillips L. Key informants' perceptions of health care for elders at the U.S.-Mexico border. Public Health Nurs. 2006; 23(3):224.) 